\title{
Smoothness and Gaussian Density Estimates for Stochastic Functional Differential Equations with Fractional Noise
}

\author{
Nguyen Van Tan* \\ Department of Foundation, Academy of Cryptography Techniques, Hanoi, Vietnam
}

\begin{abstract}
In this paper, we study the density of the solution to a class of stochastic functional differential equations driven by fractional Brownian motion. Based on the techniques of Malliavin calculus, we prove the smoothness and establish upper and lower Gaussian estimates for the density.
\end{abstract}

Keywords Stochastic functional differential equations, Density estimates, Malliavin calculus, fractional Brownian motion

AMS 2010 subject classifications 34K50, 60H07, 60H10

DOI: $10.19139 /$ soic-2310-5070-784

\section{Introduction}

In the last decade, Gaussian density estimates for the solutions of various stochastic equations have been intensively studied. Particularly, the class of stochastic equations with fractional noise has been discussed by several authors, see $[1,2,4,8]$ and references therein.

We recall that fractional Brownian motion $(\mathrm{fBm})$ of Hurst parameter $H \in(0,1)$ is a centered Gaussian process $B^{H}=\left(B_{t}^{H}\right)_{t \in \mathbb{R}_{+}}$with covariance function

$$
R_{H}(t, s):=E\left[B_{t}^{H} B_{s}^{H}\right]=\frac{1}{2}\left(t^{2 H}+s^{2 H}-|t-s|^{2 H}\right) .
$$

For $H>\frac{1}{2}, B_{t}^{H}$ admits the so-called Volterra representation

$$
B_{t}^{H}=\int_{0}^{t} K_{H}(t, s) d B_{s}
$$

where $\left(B_{t}\right)_{t \epsilon_{+}}$is a standard Brownian motion, the kernel $K_{H}$ is defined by

$$
K_{H}(t, s)=C_{H} s^{1 / 2-H} \int_{s}^{t}(u-s)^{H-\frac{3}{2}} u^{H-\frac{1}{2}} d u, \quad s \leq t
$$

with $C_{H}:=\sqrt{\frac{H(2 H-1)}{\beta(2-2 H, H-1 / 2)}}$, where $\beta$ is the Beta function.

*Correspondence to: Nguyen Van Tan (Email: hoatulip18@gmail.com). Department of Foundation, Academy of Cryptography Techniques, 141 Chien Thang, Thanh Tri, Hanoi, Vietnam.

ISSN 2310-5070 (online) ISSN 2311-004X (print)

Copyright (C) 2020 International Academic Press 
In this paper, we consider stochastic functional differential equations of the form

$$
\left\{\begin{array}{l}
X_{t}=\eta(0)+\int_{0}^{t}\left[\int_{-r}^{0} \rho(u) g\left(X_{u+s}\right) d u+a\left(s, X_{s}\right)\right] d s+\int_{0}^{t} \sigma\left(s, X_{s}\right) d B_{s}^{H}, \quad t \in[0, T], \\
X_{t}=\eta(t), \quad t \in[-r, 0],
\end{array}\right.
$$

where $r>0$ is delay time, the kernel $\rho$ and initial condition $\eta$ are deterministic functions on $[-r, 0]$. The stochastic integral is interpreted as a pathwise Riemann-Stieltjes integral, which has been frequently used in the studies related to $\mathrm{fBm}$. We refer the reader to [12] for a detailed presentation of this integral.

The density of solutions to the equation (2) has been discussed in some special cases. When $H=\frac{1}{2}, B^{H}$ reduces to standard Brownian motion and in this case, the existence and smoothness of the probability density of solutions were proved by Takeuchi in [11]. When $H>\frac{1}{2}$, Gaussian density estimates were obtained by Dung et al. in [6] for the equation (2) with $g=0$. However, the case of $g \neq 0$ has not investigated yet. Thus, in the present paper, our aim is to establish analogue results for the equation (2) with $g \neq 0$ and $H>\frac{1}{2}$. More specifically, we obtain the following properties:

(i) the existence and Gaussian estimates for the density of solutions,

(ii) the smoothness of the density of solutions.

It should be noted that the information about the density will be very useful in practical studies, see e.g. [7]. In a spirit close to $[6,11]$, the main tools of this paper are the techniques of Malliavin calculus. However, we would like to emphasize that the complexity of stochastic integrals with respect to $\mathrm{fBm}$ and the appearance of delayed integral term in (2) require a fine analysis for proving the properties (i) and (ii). The rest of this article is organized as follows. In Section 2, we recall some fundamental concepts of Malliavin calculus and a general Gaussian estimate for the density of Malliavin differentiable random variables. The main results of the paper are stated and proved in Section 3. The conclusion is given in Section 4.

\section{Preliminaries}

Let us recall some elements of Malliavin calculus with respect to Brownian motion $B$, where $B$ is used to present $B_{t}^{H}$ as in (1) (for more details see [9]). We suppose that $\left(B_{t}\right)_{t \in[0, T]}$ is defined on a complete probability space $(\Omega, \mathcal{F}, \mathbb{F}, P)$, where $\mathbb{F}=\left(\mathcal{F}_{t}\right)_{t \in[0, T]}$ is a natural filtration generated by the Brownian motion $B$. For $h \in L^{2}[0, T]$, we denote by $B(h)$ the Wiener integral

$$
B(h)=\int_{0}^{T} h(t) d B_{t} .
$$

Let $\mathcal{S}$ denote the dense subset of $L^{2}(\Omega, \mathcal{F}, P)$ consisting of smooth random variables of the form

$$
F=f\left(B\left(h_{1}\right), \ldots, B\left(h_{n}\right)\right),
$$

where $n \in \mathbb{N}, f \in C_{b}^{\infty}\left({ }^{n}\right), h_{1}, \ldots, h_{n} \in L^{2}[0, T]$. If $F$ has the form (3), we define its Malliavin derivative as the process $D F:=\left\{D_{t} F, t \in[0, T]\right\}$ given by

$$
D_{t} F=\sum_{k=1}^{n} \frac{\partial f}{\partial x_{k}}\left(B\left(h_{1}\right), \ldots, B\left(h_{n}\right)\right) h_{k}(t) .
$$

More generally, we can define the $k$ th order derivative $D^{k} F$ by iterating the derivative operator $k$ times, i.e. $D_{t_{1}, \ldots, t_{k}}^{k} F=D_{t_{k}} \ldots D_{t_{1}} F$. For any integer $k$ and any $p \geq 1$, we denote by $D^{k, p}$ the closure of $\mathcal{S}$ with respect to the norm

$$
\|F\|_{k, p}^{p}:=E|F|^{p}+E\left[\int_{0}^{T}\left|D_{u_{1}} F\right|^{p} d u_{1}\right]+E\left[\int_{0}^{T} \ldots \int_{0}^{T}\left|D_{u_{1}, \ldots, u_{k}}^{k} F\right|^{p} d u_{1} \ldots . d u_{k}\right] .
$$


A random variable $F$ is said to be Malliavin differentiable if it belongs to $\mathbb{D}^{1,2}$.

In order to obtain Gaussian density estimates for solutions to the equation (2), we will use a general criterion established recently by Nourdin and Viens in [10]. We recall here [6, Theorem 2.4] for a convenient version which can be of interest for the readers who are not used to working with the Ornstein-Uhlenbeck operator.

Proposition 1

Let $\mathrm{F}$ be in $\mathbb{D}^{1,2}$ with mean zero. If there exist positive constants $\mathrm{c}, \mathrm{C}$ such that, for all $x \in$, almost surely

$$
c \leq \int_{0}^{T} D_{r} F E\left[D_{r} F \mid \mathcal{F}_{r}\right] d r \leq C,
$$

then the density $\rho_{F}$ of $\mathrm{F}$ exists and satisfies, for almost all $x \in$

$$
\frac{E|F|}{2 C} \exp \left(-\frac{x^{2}}{2 c}\right) \leq \rho_{F}(x) \leq \frac{E|F|}{2 c} \exp \left(-\frac{x^{2}}{2 C}\right)
$$

\section{The main results}

In the whole this section, we consider the equation (2) with the following fundamental assumptions. Note that the conditions on $a$ and $\sigma$ are similar to that required in Section 5 of [6].

$\left(A_{1}\right)$ The coefficients $a, g, \sigma \in \mathcal{C}_{b}^{1,1}([0, T] \times)$, and there exists a constant $m>0$ so that $|\sigma(t, x)| \geq m$, for all $(t, x) \in[0, T] \times$.

$\left(A_{2}\right)$ The kernel $\rho:[0, T] \rightarrow$ satisfies:

$$
\int_{0}^{T}|\rho(s)| d s<\infty .
$$

Let us first give a short discussion about the existence and uniqueness of solutions. We denote by $\mathcal{C}_{b}^{1,1}([0, T] \times)$ the space of bounded functions $f:[0, T] \times \mathbb{R} \rightarrow \mathbb{R}$ with bounded partial derivatives of the first order and we write

$$
f_{1}^{\prime}(s, x)=\frac{\partial f(s, x)}{\partial s}, \quad f_{2}^{\prime}(s, x)=\frac{\partial f(s, x)}{\partial x} .
$$

We define the function

$$
F(t, x):=\int_{0}^{x} \frac{1}{\sigma(t, u)} d u, \quad(t, x) \in[0, T] \times \mathbb{R} .
$$

For $(t, z) \in[0, T] \times \mathbb{R}$, consider the function $\Phi(t, z):=F(t, z)-x$, where $x \in \mathbb{R}$ is fixed. Since $\Phi_{2}^{\prime}(t, z)=$ $\sigma(t, z)^{-1} \neq 0$, by the Implicit Function Theorem, there exists a function $\bar{G}(t, x)$ such as $\Phi(t, \bar{G}(t, x))=0$, i.e. $F(t, \bar{G}(t, x))=x$. Moreover, we have

$$
\begin{aligned}
& F_{2}^{\prime}(t, x)=\sigma(t, x)^{-1} \quad \text { and } \quad F_{1}^{\prime}(t, x)=-\int_{0}^{x} \frac{\sigma_{1}^{\prime}(t, u)}{(\sigma(t, u))^{2}} d u, \\
& \bar{G}_{2}^{\prime}(t, x)=\left(F_{2}^{\prime}(t, \bar{G}(t, x))\right)^{-1}=\sigma(t, \bar{G}(t, x)), \\
& \bar{G}_{1}^{\prime}(t, x)=-\frac{F_{1}^{\prime}(t, \bar{G}(t, x))}{F_{2}^{\prime}(t, \bar{G}(t, x))}=-F_{1}^{\prime}(t, \bar{G}(t, x)) \sigma(t, \bar{G}(t, x)) .
\end{aligned}
$$

Set $G(t, x)$ defined by

$$
\left\{\begin{array}{l}
G(t, x):=\bar{G}(t, x) \quad t \in[0, T] \\
G(t, x)=\eta(t) \quad t \in[-r, 0]
\end{array}\right.
$$


We consider stochastic functional differential equation with additive noise

$$
Y_{t}=y_{0}+\int_{0}^{t}\left(A\left(s, Y_{s}\right)+\frac{1}{\sigma\left(s, G\left(s, Y_{s}\right)\right)} \int_{s-r}^{s} \rho(u-s) g\left(u, G\left(u, Y_{u}\right)\right) d u\right) d s+B_{t}^{H},
$$

where $y_{0}:=F\left(0, x_{0}\right)$, and $A(y, s)=F_{1}^{\prime}(s, G(s, y))+\frac{a(s, G(s, y)}{\sigma(s, G(s, y))}$.

It was already pointed out in [6] that $A(y, s)$ is Lipschitz and has linear growth. On the other hand, under Assumptions $\left(A_{1}\right)$ and $\left(A_{2}\right)$, we can check that the functions $\frac{1}{\sigma(s, G(s, y))}$ and $g(s, G(s, y))$ are also Lipschitz and have linear growth. Hence, by repeating the computations presented in the proof of Proposition 3.1 in [3], we can infer that the equation (7) admits a unique strong solution $\left(Y_{t}\right)_{t \in[0, T]}$.

Based on the properties of $\left(Y_{t}\right)_{t \in[0, T]}$, we have the following propositions.

\section{Proposition 2}

Let Assumptions $\left(A_{1}\right)$ and $\left(A_{2}\right)$ hold. Then, the equation (2) has a unique strong solution given by $X_{t}=$ $G\left(t, Y_{t}\right),-r \leq t \leq T$. This solution is an $\mathcal{F}_{t}$-adapted process and, for all $\varepsilon \in(0, H)$, whose trajectories are Hölder continuous of order $H-\varepsilon$ on $[0, T]$.

\section{Proof}

The proof is similar to that of Lemma 5.1 in [6]. So we omit it.

\section{Proposition 3}

Under the Assumptions $\left(A_{1}\right)$ and $\left(A_{2}\right)$, the unique strong solution $\left(X_{t}\right)_{t \in[-r, T]}$ to the equation (2) is Malliavin differentiable and satisfies $D_{\theta} X_{t}=0$ for $\theta>t$ or $t \in[-r, 0]$, and for all $0 \leq \theta \leq t \leq T$,

$$
D_{\theta} X_{t}=\sigma\left(t, X_{t}\right)\left(\int_{\theta}^{t} N\left(s, X_{s}\right) D_{\theta} X_{s} d s+\int_{\theta}^{t} \frac{1}{\sigma\left(s, X_{s}\right)} \int_{s-r}^{s} \rho(u-s) g_{2}^{\prime}\left(u, X_{u}\right) D_{\theta} X_{u} d u d s+K_{H}(t, \theta)\right),
$$

where

$$
N\left(s, X_{s}\right):=-\frac{\sigma_{1}^{\prime}\left(s, X_{s}\right)}{\sigma^{2}\left(s, X_{s}\right)}-\frac{\sigma_{2}^{\prime}\left(s, X_{s}\right)}{\sigma^{2}\left(s, X_{s}\right)} \int_{s-r}^{s} \rho(u-s) g\left(u, X_{u}\right) d u+\frac{a_{2}^{\prime}\left(s, X_{s}\right) \sigma\left(s, X_{s}\right)-a\left(s, X_{s}\right) \sigma_{2}^{\prime}\left(s, X_{s}\right)}{\sigma^{2}\left(s, X_{s}\right)} .
$$

Proof

Let $\left(Y_{t}\right)_{t \in[0, T]}$ be the unique strong solution to (7). By using the same argument as in the proof of Lemma 5.3 in [6], we have $Y_{t} \in \mathbb{D}^{1,2}$ and its Malliavin derivative is given by

$$
\begin{aligned}
D_{\theta} Y_{t}=\mathbf{1}_{[0, t]}(\theta) & \left(\int_{\theta}^{t} M\left(s, Y_{s}\right) D_{\theta} Y_{s} d s+K_{H}(t, \theta)\right. \\
& \left.+\int_{\theta}^{t} \frac{1}{\sigma\left(s, G\left(s, Y_{s}\right)\right)} \int_{s-r}^{s} \rho(u-s) g_{2}^{\prime}\left(u, G\left(u, Y_{u}\right)\right) \sigma\left(u, G\left(u, Y_{u}\right)\right) D_{\theta} Y_{u} d u d s\right), 0 \leq t \leq T
\end{aligned}
$$

where

$$
\begin{aligned}
M\left(s, Y_{s}\right):=-\frac{\sigma_{1}^{\prime}\left(s, G\left(s, Y_{s}\right)\right)}{\sigma\left(s, G\left(s, Y_{s}\right)\right)}-\frac{\sigma_{2}^{\prime}\left(s, G\left(s, Y_{s}\right)\right)}{\sigma\left(s, G\left(s, Y_{s}\right)\right)} \int_{s-r}^{s} \rho(u-s) g\left(u, G\left(u, Y_{u}\right)\right) d u \\
\quad+\frac{a_{2}^{\prime}\left(s, G\left(s, Y_{s}\right)\right) \sigma\left(s, G\left(s, Y_{s}\right)\right)-a\left(s, G\left(s, Y_{s}\right)\right) \sigma_{2}^{\prime}\left(s, G\left(s, Y_{s}\right)\right)}{\sigma\left(s, G\left(s, Y_{s}\right)\right)} .
\end{aligned}
$$


From the relation $X_{t}=G\left(t, Y_{t}\right)$ and the chain rule of Malliavin derivatives (see Proposition 1.2.3 in [9]), we have $X_{t} \in \mathbb{D}^{1,2}$, and

$$
D_{\theta} X_{t}=G_{2}^{\prime}\left(t, Y_{t}\right) D_{\theta} Y_{t}=\sigma\left(t, X_{t}\right) D_{\theta} Y_{t}, \quad 0 \leq \theta \leq t \leq T .
$$

This, combined with (9), gives us (8). So the proof of Proposition is complete.

From now on, we will use the symbol $C$ to denote a generic constant, whose value may change from one line to another.

Proposition 4

Let $\left(X_{t}\right)_{t \in[-r, T]}$ be the solution to the equation (2). Assume that $\left(A_{1}\right),\left(A_{2}\right)$ hold. Then there exists a finite constant $C>0$ such that:

$$
\left|D_{\theta} X_{t}\right| \leq C K_{H}(t, \theta), \quad \text { a.s. }
$$

for all $0 \leq \theta \leq t \leq T$.

Proof

From (9), $\left(A_{1}\right),\left(A_{2}\right)$ and the boundedness of $A\left(t, Y_{t}\right)$, we have

$$
\begin{aligned}
\left|D_{\theta} Y_{t}\right| & \leq \int_{\theta}^{t}\left|M\left(s, Y_{s}\right)\right|\left|D_{\theta} Y_{s}\right| d s+C \int_{\theta}^{t}\left|D_{\theta} Y_{s}\right| d s+K_{H}(t, \theta) \\
& \leq C \int_{\theta}^{t}\left|D_{\theta} Y_{s}\right| d s+K_{H}(t, \theta), \quad \text { for all } 0 \leq \theta \leq t \leq T .
\end{aligned}
$$

An application of Gronwall's inequality now gives that

$$
\left|D_{\theta} Y_{t}\right| \leq K_{H}(t, \theta)+C \int_{\theta}^{t} K_{H}(s, \theta) \exp \{C(t-s)\} d s \leq K_{H}(t, \theta)+C \int_{\theta}^{t} K_{H}(s, \theta) d s .
$$

Since $\partial_{1} K_{H}(t, \theta) \geq 0$, we have

$$
\left|D_{\theta} Y_{t}\right| \leq K_{H}(t, \theta)+C(t-\theta) K_{H}(t, \theta) \leq C K_{H}(t, \theta) .
$$

From (10), (12) and the boundedness of $\sigma(t, x)$, we have

$$
\left|D_{\theta} X_{t}\right| \leq C\left|\sigma\left(t, X_{t}\right)\right| K_{H}(t, \theta) \leq C K_{H}(t, \theta), \quad \text { for all } 0 \leq \theta \leq t \leq T .
$$

\section{Proposition 5}

Let $\left(X_{t}\right)_{t \in[-r, T]}$ be the solution to the equation (2). Assume that $\left(A_{1}\right),\left(A_{2}\right)$ hold. Then there exists a finite constant $c>0$ such that

$$
\int_{0}^{t} D_{\theta} X_{t} E\left[D_{\theta} X_{t} \mid \mathcal{F}_{\theta}\right] d \theta \geq c t^{2 H}, \quad \text { a.s }
$$

for all $t \in(0, T]$.

Proof

It follows from (9) that $\left(D_{\theta} Y_{t}\right)_{t \in[\theta, T]}$ solves the following ordinary differential equation

$$
\begin{aligned}
& d D_{\theta} Y_{t} \\
& =\left(M\left(t, Y_{t}\right) D_{\theta} Y_{t}+\frac{1}{\sigma\left(t, G\left(s, Y_{t}\right)\right)} \int_{t-r}^{t} \rho(s-t) g_{2}^{\prime}\left(s, G\left(s, Y_{s}\right)\right) \sigma\left(s, G\left(s, Y_{s}\right)\right) D_{\theta} Y_{s} d s\right) d t+\partial_{1} K_{H}(t, \theta) d t,
\end{aligned}
$$


with the initial condition $\left.D_{\theta} Y_{t}\right|_{t=\theta}=0$, where $\partial_{1} K_{H}(t, \theta)=\frac{\partial K_{H}(t, \theta)}{\partial t}$. By the Comparison Theorem and $\partial_{1} K_{H}(t, \theta) \geq 0$, it is easy that $D_{\theta} Y_{t} \geq 0$. So we have that $D_{\theta} X_{t} \geq 0$ when $\sigma(t, x)>0$ and $D_{\theta} X_{t} \leq 0$ when $\sigma(t, x)<0$. Hence, for all $0 \leq \theta \leq t \leq T$,

$$
D_{\theta} X_{t} E\left[D_{\theta} X_{t} \mid \mathcal{F}_{\theta}\right] \geq 0 \quad \text { a.s. }
$$

Define

$$
h(t):=\int_{(1-\varepsilon) t}^{t} D_{\theta} X_{t} E\left[D_{\theta} X_{t} \mid \mathcal{F}_{\theta}\right] d \theta=\int_{(1-\varepsilon) t}^{t} \sigma\left(t, X_{t}\right) D_{\theta} Y_{t} E\left[\sigma\left(t, X_{t}\right) D_{\theta} Y_{t} \mid \mathcal{F}_{\theta}\right] d \theta, \quad 0<\varepsilon \leq 1 .
$$

From the equation (9), we have

$$
D_{\theta} Y_{t}=U(t, \theta)+K_{H}(t, \theta), \quad \theta \leq t .
$$

where

$$
U(t, \theta):=\int_{\theta}^{t} M\left(s, Y_{s}\right) D_{\theta} Y_{s} d s+\int_{\theta}^{t} \frac{1}{\sigma\left(s, G\left(s, Y_{s}\right)\right)} \int_{s-r}^{s} \rho(u-s) g_{2}^{\prime}\left(u, G\left(u, Y_{u}\right)\right) \sigma\left(u, G\left(u, Y_{u}\right)\right) D_{\theta} Y_{u} d u d s
$$

Thus we can rewrite $h(t)$ as follows

$$
\begin{aligned}
h(t) & =\int_{(1-\varepsilon) t}^{t} \sigma\left(t, X_{t}\right) U(t, \theta) E\left[\sigma\left(t, X_{t}\right) U(t, \theta) \mid \mathcal{F}_{\theta}\right] d \theta+\int_{(1-\varepsilon) t}^{t} \sigma\left(t, X_{t}\right) U(t, \theta) E\left[\sigma\left(t, X_{t}\right) K_{H}(t, \theta) \mid \mathcal{F}_{\theta}\right] d \theta \\
& +\int_{(1-\varepsilon) t}^{t} \sigma\left(t, X_{t}\right) K_{H}(t, \theta) E\left[\sigma\left(t, X_{t}\right) U(t, \theta) \mid \mathcal{F}_{\theta}\right] d \theta+\int_{(1-\varepsilon) t}^{t} \sigma\left(t, X_{t}\right) K_{H}(t, \theta) E\left[\sigma\left(t, X_{t}\right) K_{H}(t, \theta) \mid \mathcal{F}_{\theta}\right] d \theta
\end{aligned}
$$

From (12) and $\partial_{1} K_{H}(t, \theta) \geq 0$,

$$
|U(t, \theta)| \leq C \int_{\theta}^{t} K_{H}(s, \theta) d s \leq C K_{H}(t, \theta)(t-\theta), \quad \theta \leq t .
$$

On the other hand, for all $0 \leq s \leq t$, we have

$$
\begin{aligned}
E\left|B_{t}^{H}-B_{s}^{H}\right|^{2}= & E\left(\int_{0}^{s}\left[K_{H}(t, u)-K_{H}(s, u)\right] d B_{u}+\int_{s}^{t} K_{H}(t, u) d B_{u}\right)^{2} \\
& =E\left(\int_{0}^{s}\left[K_{H}(t, u)-K_{H}(s, u)\right] d B_{u}\right)^{2}+E\left(\int_{s}^{t} K_{H}(t, u) d B_{u}\right)^{2} \\
& \geq E\left(\int_{s}^{t} K_{H}(t, u) d B_{u}\right)^{2}=\int_{s}^{t} K_{H}^{2}(t, u) d u .
\end{aligned}
$$

Using the fact that $E\left|B_{t}^{H}-B_{s}^{H}\right|^{2}=|t-s|^{2 H}$, we deduce

$$
\int_{s}^{t} K_{H}^{2}(t, u) d u \leq|t-s|^{2 H}, 0 \leq s \leq t
$$


From (14) and (15), we can get the following estimates

$$
\begin{gathered}
\left|\int_{(1-\varepsilon) t}^{t} \sigma\left(t, X_{t}\right) U(t, \theta) E\left[\sigma\left(t, X_{t}\right) K_{H}(t, \theta) \mid \mathcal{F}_{\theta}\right] d \theta\right| \leq C \int_{(1-\varepsilon) t}^{t}(t-\theta) K_{H}^{2}(t, \theta) d \theta \\
\leq C(\varepsilon t) \int_{(1-\varepsilon) t}^{t} K_{H}^{2}(t, \theta) d \theta \\
\leq C(\varepsilon t)(\varepsilon t)^{2 H}=C(\varepsilon t)^{2 H+1} \\
\int_{(1-\varepsilon) t}^{t} \sigma\left(t, X_{t}\right) K_{H}(t, \theta) E\left[\sigma\left(t, X_{t}\right) U(t, \theta) \mid \mathcal{F}_{\theta}\right] d \theta \mid \leq C(\varepsilon t)^{2 H+1} \\
\int_{(1-\varepsilon) t}^{t} \sigma\left(t, X_{t}\right) U(t, \theta) E\left[\sigma\left(t, X_{t}\right) U(t, \theta) \mid \mathcal{F}_{\theta}\right] d \theta \mid \leq C \int_{(1-\varepsilon) t}^{t}(t-\theta)^{2} K_{H}^{2}(t, \theta) d \theta \\
\leq C(\varepsilon t)^{2} \int_{(1-\varepsilon) t}^{t} K_{H}^{2}(t, \theta) d \theta=C(\varepsilon t)^{2 H+2}
\end{gathered}
$$

From the definition of $K_{H}(t, r)$, for all $0<r \leq t$, we deduce

$$
K_{H}(t, r) \geq C_{H} \int_{r}^{t}(u-r)^{H-\frac{3}{2}} d u=\frac{C_{H}}{H-\frac{1}{2}}(t-r)^{H-\frac{1}{2}}
$$

So, for all $0<s \leq t$, we have

$$
\begin{aligned}
\int_{s}^{t} K_{H}^{2}(t, \theta) d \theta & \geq\left(\frac{C_{H}}{H-\frac{1}{2}}\right)^{2} \int_{s}^{t}(t-\theta)^{2 H-1} d \theta \\
& =\frac{1}{2 H}\left(\frac{C_{H}}{H-\frac{1}{2}}\right)^{2}(t-s)^{2 H} \\
& =C_{H}^{\prime}(t-s)^{2 H}
\end{aligned}
$$

where $C_{H}^{\prime}:=\frac{1}{2 H}\left(\frac{C_{H}}{H-\frac{1}{2}}\right)^{2}$. Making use of the elementary inequality $|a+b| \geq|a|-|b|$ yields

$$
\begin{aligned}
h(t) & \geq\left|\int_{(1-\varepsilon) t}^{t} \sigma\left(t, X_{t}\right) K_{H}(t, \theta) E\left[\sigma\left(t, X_{t}\right) K_{H}(t, \theta) \mid \mathcal{F}_{\theta}\right] d \theta\right|-2 C(\varepsilon t)^{2 H+1}-C(\varepsilon t)^{2 H+2} \\
& \geq(\varepsilon t)^{2 H}\left(C_{H}^{\prime} m^{2}-2 C(\varepsilon t)-C(\varepsilon t)^{2}\right) .
\end{aligned}
$$

Now we choose $\varepsilon \in(0,1]$ such that

$$
C(\varepsilon T)^{2}+2 C(\varepsilon T) \leq \frac{C_{H}^{\prime} m^{2}}{2}
$$


Then, we get

$$
h(t) \geq \frac{C_{H}^{\prime} m^{2}}{2}(\varepsilon t)^{2 H}:=c t^{2 H} .
$$

So we can finish the proof of Proposition because

$$
\int_{0}^{t} D_{\theta} X_{t} E\left[D_{\theta} X_{t} \mid \mathcal{F}_{\theta}\right] d \theta \geq h(t)
$$

We now are ready to formulate and prove the main results of this paper.

\section{Theorem 1}

Asume that $\left(A_{1}\right)$ and $\left(A_{2}\right)$ hold and let $\left(X_{t}\right)_{t \in[-r, T]}$ be the unique strong solution to the equation (2). Then, for each $t \in(0, T]$, the density $\rho_{X_{t}}$ exists and satisfies the bounds for all $x \in \mathbb{R}$

$$
\frac{E\left|X_{t}-E X_{t}\right|}{2 C t^{2 H}} \exp \left(-\frac{\left(x-E X_{t}\right)^{2}}{2 c t^{2 H}}\right) \leq \rho_{X_{t}}(x) \leq \frac{E\left|X_{t}-E X_{t}\right|}{2 c t^{2 H}} \exp \left(-\frac{\left(x-E X_{t}\right)^{2}}{2 C t^{2 H}}\right) .
$$

where $\mathrm{c}, \mathrm{C}$ are finite positive constants.

Proof

For each $t \in(0, T]$, we consider the random variable $F:=X_{t}-E X_{t}$. Clearly, $F$ has mean zero and is Malliavin differentiable with $D_{\theta} F=D_{\theta} X_{t}$. Hence, by Propositions 4 and 5 , we can get

$$
0<c t^{2 H} \leq \int_{0}^{T} D_{\theta} F E\left[D_{\theta} F \mid \mathcal{F}_{\theta}\right] d \theta=\int_{0}^{t} D_{\theta} X_{t} E\left[D_{\theta} X_{t} \mid \mathcal{F}_{\theta}\right] d \theta \leq C t^{2 H},
$$

where c, $\mathrm{C}$ are some finite positive constants. In view of Proposition 1, we can conclude that the density $\rho_{F}$ of the random variable $F$ exits and satisfies

$$
\frac{E|F|}{2 C t^{2 H}} \exp \left(-\frac{x^{2}}{2 c t^{2 H}}\right) \leq \rho_{F}(x) \leq \frac{E|F|}{2 c t^{2 H}} \exp \left(-\frac{x^{2}}{2 C t^{2 H}}\right), x \in \mathbb{R},
$$

which gives us (17) because $\rho_{X_{t}}(x)=\rho_{F}\left(x-E X_{t}\right)$.

\section{Theorem 2}

Suppose the Assumptions $\left(A_{1}\right)$ and $\left(A_{2}\right)$. Let $\left(X_{t}\right)_{t \in[-r, T]}$ be the solution to the equation (2). In addition, we assume that $a, g$ and $\sigma$ are infinitely differentiable functions in $x$ with bounded derivatives of all orders. Then, for each $t \in(0, T]$, the random variable $X_{t}$ has an infinitely differentiable density with respect to Lebesgue measure on $\mathbb{R}$.

\section{Proof}

Fix $t \in(0, T]$, thanks to Theorem 2.1.4 in [9], we have to check the following properties

(a) $X_{t} \in \mathbb{D}^{\infty}=\bigcap_{i \geq 1} \bigcap_{p \geq 1} \mathbb{D}^{i, p}$,

(b) $\left(\int_{0}^{t}\left|D_{\theta} X_{t}\right|^{2} d \theta\right)^{-1} \in \bigcap_{p \geq 1} L^{p}(\Omega)$. 
It is easy to verify that the coefficients of the equation (7) are infinitely differentiable in $y$ with bounded derivatives of all orders. Hence, we can infer that $Y_{t} \in \mathbb{D}^{\infty}$. So $X_{t}$ does. Let us now check the property $(b)$.

By Proposition 3, we have

$$
D_{\theta} X_{t}=\sigma\left(t, X_{t}\right)\left(\int_{\theta}^{t} N\left(s, X_{s}\right) D_{\theta} X_{s} d s+\int_{\theta}^{t} \frac{1}{\sigma\left(s, X_{s}\right)} \int_{s-r}^{s} \rho(u-s) g_{2}^{\prime}\left(u, X_{u}\right) D_{\theta} X_{u} d u d s+K_{H}(t, \theta)\right) .
$$

Hence,

$$
\begin{aligned}
& \int_{0}^{t}\left|D_{\theta} X_{t}\right|^{2} d \theta \\
& =\int_{0}^{t}\left(\sigma\left(t, X_{t}\right)\left(\int_{\theta}^{t} N\left(s, X_{s}\right) D_{\theta} X_{s} d s+\int_{\theta}^{t} \frac{1}{\sigma\left(s, X_{s}\right)} \int_{s-r}^{s} \rho(u-s) g_{2}^{\prime}\left(u, X_{u}\right) D_{\theta} X_{u} d u d s+K_{H}(t, \theta)\right)\right)^{2} d \theta .
\end{aligned}
$$

For each $y \geq y_{0}:=\frac{4}{C_{H}^{\prime} t^{2 H} m^{2}}$, where $C_{H}^{\prime}=\frac{1}{2 H}\left(\frac{C_{H}}{H-\frac{1}{2}}\right)^{2}$, the real number $\varepsilon:=\left(\frac{4}{y C_{H}^{\prime} m^{2} t^{2 H}}\right)^{\frac{1}{2 H}}$ belongs to $(0,1]$. Using the fundamental inequality $(a+b+c)^{2} \geq \frac{a^{2}}{2}-2\left(b^{2}+c^{2}\right)$ and (16) we obtain

$$
\begin{aligned}
\int_{0}^{t}\left|D_{\theta} X_{t}\right|^{2} d \theta & \geq \int_{t(1-\varepsilon)}^{t} \frac{\sigma^{2}\left(t, X_{t}\right) K_{H}^{2}(t, \theta)}{2} d \theta-2 \int_{t(1-\varepsilon)}^{t}\left(\sigma\left(t, X_{t}\right) \int_{\theta}^{t} N\left(s, X_{s}\right) D_{\theta} X_{s} d s\right)^{2} d \theta \\
& -2 \int_{t(1-\varepsilon)}^{t}\left(\sigma\left(t, X_{t}\right) \int_{\theta}^{t} \frac{1}{\sigma\left(s, X_{s}\right)} \int_{s-r}^{s} \rho(u-s) g_{2}^{\prime}\left(u, X_{u}\right) D_{\theta} X_{u} d u d s\right)^{2} d \theta \\
& \geq \frac{C_{H}^{\prime} m^{2}(\varepsilon t)^{2 H}}{2}-I_{y}(t) \\
& =\frac{2}{y}-I_{y}(t),
\end{aligned}
$$

where

$$
\begin{aligned}
I_{y}(t) & =2 \int_{t(1-\varepsilon)}^{t}\left(\sigma\left(t, X_{t}\right) \int_{\theta}^{t} N\left(s, X_{s}\right) D_{\theta} X_{s} d s\right)^{2} d \theta \\
& +2 \int_{t(1-\varepsilon)}^{t}\left(\sigma\left(t, X_{t}\right) \int_{\theta}^{t} \frac{1}{\sigma\left(s, X_{s}\right)} \int_{s-r}^{s} \rho(u-s) g_{2}^{\prime}\left(u, X_{u}\right) D_{\theta} X_{u} d u d s\right)^{2} d \theta
\end{aligned}
$$

By Markov's inequality, we have

$$
P\left(\int_{0}^{t}\left|D_{\theta} X_{t}\right|^{2} d \theta \leq \frac{1}{y}\right) \leq P\left(\frac{2}{y}-I_{y}(t) \leq \frac{1}{y}\right)=P\left(I_{y}(t) \geq \frac{1}{y}\right) \leq y^{p / 2} E\left(\left|I_{y}(t)\right|^{p / 2}\right) \quad \forall p \geq 2 .
$$


Under the assumptions $\left(A_{1}\right),\left(A_{2}\right)$ and the inequality $(|a|+|b|)^{p / 2} \leq 2^{p / 2-1}\left(|a|^{p / 2}+|b|^{p / 2}\right)$, we can get

$$
\begin{aligned}
E\left|I_{y}(t)\right|^{p / 2} & =2^{p / 2} E\left(\int_{t(1-\varepsilon)}^{t}\left(\sigma\left(t, X_{t}\right) \int_{\theta}^{t} N\left(s, X_{s}\right) D_{\theta} X_{s} d s\right)^{2} d \theta\right. \\
& \left.+\int_{t(1-\varepsilon)}^{t}\left(\sigma\left(t, X_{t}\right) \int_{\theta}^{t} \frac{1}{\sigma\left(s, X_{s}\right)} \int_{s-r}^{s} \rho(u-s) g_{2}^{\prime}\left(u, X_{u}\right) D_{\theta} X_{u} d u d s\right)^{2} d \theta\right)^{p / 2} \\
& \leq 2^{p-1} E\left(\int_{t(1-\varepsilon)}^{t}\left(\sigma\left(t, X_{t}\right) \int_{\theta}^{t} N\left(s, X_{s}\right) D_{\theta} X_{s} d s\right)^{2} d \theta\right)^{p / 2} \\
& +2^{p-1} E\left(\int_{t(1-\varepsilon)}^{t}\left(\sigma\left(t, X_{t}\right) \int_{\theta}^{t} \frac{1}{\sigma\left(s, X_{s}\right)} \int_{s-r}^{s} \rho(u-s) g_{2}^{\prime}\left(u, X_{u}\right) D_{\theta} X_{u} d u d s\right)^{2} d \theta\right)^{p / 2} \\
& \leq 2^{p-1} E\left(\left(C \int_{t(1-\varepsilon)}^{t}\left(\int_{\theta}^{t} D_{\theta} X_{s} d s\right)^{2} d \theta\right)^{p / 2}+\left(C \int_{t(1-\varepsilon)}^{t}\left(\int_{\theta}^{t} \int_{s-r}^{s} \rho(u-s) D_{\theta} X_{u} d u d s\right)^{2} d \theta\right)^{p / 2}\right) \\
& \left.\leq C E\left(\left(\left.\int_{t(1-\varepsilon)}^{t}\left|\int_{\theta}^{t}\right| D_{\theta} X_{s}\right|^{2} d s d \theta\right)^{p / 2}+\left(\int_{t(1-\varepsilon)}^{t} \rho(u-s) D_{\theta} X_{u} d u d s\right)^{2} d \theta\right)^{p / 2}\right)^{p}
\end{aligned}
$$

where $C$ is some positive constant. By using Hölder's inequality we obtain

$$
\begin{aligned}
\left(\int_{\theta}^{t} \int_{s-r}^{s} \rho(u-s) D_{\theta} X_{u} d u d s\right)^{2} & \leq(t-\theta) \int_{\theta}^{t}\left(\int_{s-r}^{s}\left|\rho(u-s) D_{\theta} X_{u}\right| d u\right)^{2} d s \\
& \leq T \int_{\theta}^{t}\left(\int_{s-r}^{s}|\rho(u-s)| d u\right)\left(\int_{s-r}^{s}|\rho(u-s)|\left|D_{\theta} X_{u}\right|^{2} d u\right) d s \\
& \leq T\left(\int_{-r}^{0}|\rho(u)| d u\right)^{2} \int_{\theta}^{t}\left|D_{\theta} X_{u}\right|^{2} d u .
\end{aligned}
$$

So it holds that

$$
E\left|I_{y}(t)\right|^{\frac{p}{2}} \leq C E\left(\int_{t(1-\varepsilon)}^{t} \int_{\theta}^{t}\left|D_{\theta} X_{s}\right|^{2} d s d \theta\right)^{p / 2} \forall p \geq 2 .
$$


Using Proposition 4 and (15), we can verify that

$$
\begin{aligned}
E\left|I_{y}(t)\right|^{p / 2} \leq C E\left(\int_{t(1-\varepsilon)}^{t} \int_{\theta}^{t} K_{H}^{2}(s, \theta) d s d \theta\right)^{p / 2}=C E\left(\int_{t(1-\varepsilon)}^{t} \int_{\theta}^{t} K_{H}^{2}(s, \theta) d s d \theta\right)^{p / 2} \\
=C E\left(\int_{t(1-\varepsilon)}^{t} \int_{t(1-\varepsilon)}^{s} K_{H}^{2}(s, \theta) d \theta d s\right)^{p / 2} \leq C E\left(\int_{t(1-\varepsilon)}^{t}(s-t(1-\varepsilon))^{2 H} d s\right)^{p / 2} \\
=\frac{C}{2 H+1}(\varepsilon t)^{p(2 H+1) / 2}=C\left(\frac{4}{y C_{H}^{\prime} m^{2}}\right)^{\frac{p(2 H+1)}{4 H}}=C\left(\frac{4}{y m^{2}}\right)^{\frac{p(2 H+1)}{4 H}} \forall p \geq 2 .
\end{aligned}
$$

From (18) and (19), we deduce

$$
P\left(\int_{0}^{t}\left|D_{\theta} X_{t}\right|^{2} d \theta \leq \frac{1}{y}\right) \leq C y^{p / 2}\left(\frac{4}{y m^{2}}\right)^{\frac{p(2 H+1)}{4 H}} \forall p \geq 2 .
$$

Now for any $\alpha \geq 1$ and $p>4 H \alpha$, we have the following estimates

$$
\begin{aligned}
E\left(\int_{0}^{t}\left|D_{\theta} X_{t}\right|^{2} d \theta\right)^{-\alpha} & =\int_{0}^{\infty} \alpha y^{\alpha-1} P\left(\int_{0}^{t}\left|D_{\theta} X_{t}\right|^{2} d \theta \leq \frac{1}{y}\right) d y \\
& \leq \int_{0}^{y_{0}} \alpha y^{\alpha-1} d y+\int_{y_{0}}^{\infty} \alpha y^{\alpha-1} P\left(\int_{0}^{t}\left|D_{\theta} X_{t}\right|^{2} d \theta<\frac{1}{y}\right) d y \\
& \leq y_{0}^{\alpha}+\alpha C \int_{y_{0}}^{\infty} y^{\alpha-1} y^{p / 2}\left(\frac{4}{y m^{2}}\right)^{\frac{p(2 H+1)}{4 H}} d y \\
& =y_{0}^{\alpha}+\alpha C\left(\frac{4}{m^{2}}\right)^{\frac{p(2 H+1)}{4 H}} \frac{y_{0}^{\alpha-\frac{p}{4 H}}}{\frac{p}{4 H}-\alpha}
\end{aligned}
$$

Recalling $y_{0}=\frac{4}{C_{H}^{\prime} t^{2 H} m^{2}}$, we conclude that

$$
E\left(\int_{0}^{t}\left|D_{\theta} X_{t}\right|^{2} d \theta\right)^{-\alpha}<\infty \forall \alpha \geq 1
$$

So the property $(b)$ is proved. This finishes the proof of Theorem.

\section{Conclusion}

In this paper, we employed the techniques of Malliavin calculus to obtain smoothness and Gaussian density estimates for solutions to a fundamental class of stochastic functional differential equations with fractional noise. Our results develop further the studies initated in $[6,11]$ and hence, our work partly enriches the knowledge of the theory of stochastic functional differential equations. 
Acknowledgments. This research was funded by the Vietnam National University, Hanoi under grant number QG.20.21.

\section{REFERENCES}

1. F. Baudoin, C. Ouyang, S. Tindel, Upper bounds for the density of solutions to stochastic differential equations driven by fractional Brownian motions. Ann. Inst. Henri Poincaré Probab. Stat. 50 (2014), no. 1, 111-135.

2. M. Besalú, A. Kohatsu-Higa, S. Tindel, Gaussian-type lower bounds for the density of solutions of SDEs driven by fractional Brownian motions. Ann. Probab. 44 (2016), no. 1, 399-443.

3. N.T. Dung, Linear multifractional stochastic Volterra integro-differential equations, Taiwanese J. Math. 17 (1) (2013) 333-350.

4. N.T. Dung, The density of solutions to multifractional stochastic Volterra integro-differential equations, Nonlinear Anal. 130 (2016), 176-189.

5. N.T. Dung, Gaussian density estimates for the solution of singular stochastic Riccati equations. Appl. Math. 61 (2016), no. 5, 515-526.

6. N.T. Dung, N. Privault, G.L. Torrisi, Gaussian estimates for the solutions of some one-dimensional stochastic equations. Potential Anal (2015) 43:289-311.

7. S. Infante, L. Sánchez, A. Hernández, Stochastic models to estimate population dynamics. Stat. Optim. Inf. Comput. 7 (2019), no. 2 , 311-328.

8. J. Liu, Ciprian A. Tudor, Analysis of the density of the solution to a semilinear SPDE with fractional noise. Stochastics 88 (2016), no. 7, 959-979.

9. D. Nualart, The Malliavin calculus and related topics. Probability and its Applications. Springer-Verlag, Berlin, second edition, 2006.

10. I. Nourdin and F.G. Viens, Density formula and concentration inequalities with Malliavin calculus, Electron. J. Probab., 14:2287$2309,2009$.

11. A. Takeuchi, Malliavin calculus for degenerate stochastic functional differential equations. Acta Appl. Math. 97 (2007), no. 1-3, 281-295.

12. M. Zähle, Integration with respect to fractal functions and stochastic calculus. I. Probab. Theory Related Fields, 111:333-374, 1998. 